\title{
CHARACTER THEORY OF INFINITE WREATH PRODUCTS
}

\author{
ROBERT BOYER
}

Received 31 August 2003 and in revised form 19 March 2005

The representation theory of infinite wreath product groups is developed by means of the relationship between their group algebras and conjugacy classes with those of the infinite symmetric group. Further, since these groups are inductive limits of finite groups, their finite characters can be classified as limits of normalized irreducible characters of prelimit finite groups. This identification is called the "asymptotic character formula." The $K_{0}$ invariant of the group $C^{\star}$-algebra is also determined.

\section{Preliminaries}

The purpose of this paper is to develop the representation theory of infinite wreath product groups (defined in Section 2) by exploiting the relationship between their group algebras and conjugacy classes with those of the infinite symmetric group [16]. Furthermore, since these groups are not type $\mathrm{I}$, that is, the unitary dual of these groups is not standard Borel space, their character theory and group $C^{\star}$-algebra play a special role in their representation theory. For instance, among the substitutes for the group dual are the space of all finite characters and the space of primitive ideals of group $C^{\star}$-algebra. Both these spaces are standard Borel spaces, so they can be effectively parameterized. Drawing attention away from irreducible representations to factor representations with characters allows the development of harmonic analysis for groups that are not type I.

The infinite wreath product groups are inductive limits of finite groups, so that their $C^{\star}$-group algebra is, in fact, an AF-algebra; that is, an inductive limit of finite-dimensional $C^{\star}$-algebras. Such algebras have been well studied by means of their $K_{0}$-group invariant. For the wreath product groups, the $K_{0}$-group has an order structure which is determined through the evaluation of both the finite and semifinite characters of the $C^{\star}$ algebra as well as a natural multiplication which makes the $K_{0}$-invariant into a special ordered ring, namely, a Riesz ring (see Section 4). Another special feature for infinite wreath products is that their finite characters can be described as limits of normalized irreducible characters of the prelimit groups which is sometimes called the "asymptotic character formula." The major principle of this paper is that all these important features of the representation theory of wreath products can be reduced to the known results for 
the infinite symmetric group. An analogous reduction was found for the infinite unitary, symplectic, and orthogonal groups. See [5] for recent applications of character theory of inductive limits of classical groups to harmonic analysis and probability theory. See [10] for an application of the asymptotic character formula to the study of multiplicities of specific representations.

We fix some notation concerning Young diagrams. A Young diagram $Y$ is a finite ideal of $\left(\mathbb{Z}^{+}\right)^{2}$ relative to the usual product order structure. Let $|Y|$ denote the number of points in the diagram; while $r_{i}(Y)$ denotes the length of its $i$ th row and $r_{\#}(Y)$ denotes the number of nonzero rows; $c_{i}(Y)$ denots its $i$ th column. Finally, we denote the Frobenius coordinates of $Y$ by

$$
\left(h_{1}(Y), \ldots, h_{d_{\#}(Y)}(Y) \mid v_{1}(Y), \ldots, v_{d_{*}(Y)}(Y)\right)
$$

where $h_{j}(Y)$, respectively, $v_{j}(Y)$, is the (horizontal, resp., vertical) coordinate $r_{j}(Y)-j$, respectively, $c_{j}(Y)-j$, (length measured from the main diagonal). $d_{\#}(Y)$ denotes the length of the main diagonal of the diagram $Y$.

Let $\mathscr{Y}_{n}$ denote the set of all Young diagrams with exactly $n$ nodes, and let $\mathscr{Y}=\bigcup_{n=0}^{\infty} \mathscr{Y}_{n}$.

An infinite Young diagram $Y$ is an infinite ideal of $\left(\mathbb{Z}^{+}\right)^{2}$. In addition to describing an infinite diagram $Y$ with its row and column lengths, $Y$ can also be specified relative to a rectangular piece together with a finite diagram. Let

$$
\mathscr{I}_{k, \ell}=\{(i, j): i \leq k \text { or } j \leq \ell\},
$$

with $k+\ell \geq 1$. Then any infinite Young diagram $Y$ with finitely many rows and columns has the form

$$
Y=\Phi_{k, \ell} \cup\left(Y_{0}+(k, \ell)\right),
$$

where $Y_{0}$ is a finite (possibly empty) Young diagram and $Y_{0}+(k, \ell)$ means the usual translate of the Young diagram by the vector $(k, \ell)$.

As a convention, for a finite group $G$ with dual space $\hat{G}$ of equivalence classes of irreducible representations, let $p(\pi)$ denote the corresponding central projection in $C^{\star}(G)$ while $e(\pi)$ denotes a minimal subprojection of $p(\pi)$. When $G$ is abelian, we may enumerate its dual as $\left\{\omega_{1}, \omega_{2}, \ldots, \omega_{|G|}\right\}$ when convenient.

\section{Algebraic structure of $K_{0}(S(G))$}

For a finite group $G$, let $S_{n}(G)$ denote its wreath product which is the canonical semidirect product of $G^{n}=G \times G \times \cdots \times G$ ( $n$ factors) where $S(n)$ acts on $G^{n}$ by permuting components. Write elements of $S_{n}(G)$ as $\left(\sigma ; g_{1}, \ldots, g_{n}\right)$ where $\sigma \in S(n)$ and $g_{1}, \ldots, g_{n} \in G$. Given a set $S$, let $F_{n}(S, Y)$ denote the set of all functions $f$ from $S$ into $y$ such that $\sum_{s \in S}|f(s)|=n$.

If $R\left(S_{n}(G)\right)$ denotes the standard additive group of representations of $S_{n}(G)$, then $\mathscr{R}=$ $\bigoplus_{n=0}^{\infty} R\left(S_{n}(G)\right)$ is a commutative graded ring with multiplication given by an induction product

$$
\pi_{1} \circ \pi_{2}=\operatorname{Ind}_{S_{m}(G) \times S_{n}(G)}^{S_{m+n}(G)}\left(\pi_{1} \times \pi_{2}\right),
$$


where $\pi_{1} \in R\left(S_{m}(G)\right)$ and $\pi_{2} \in R\left(S_{n}(G)\right)$. Let $\mathscr{R}_{0}=\bigoplus_{n=0}^{\infty} R(S(n))$ denote the corresponding graded commutative ring for the symmetric groups. In [16], canonical isomorphisms $T_{\omega}$, for each $\omega \in \hat{G}$, are described from $\mathscr{R}_{0}$ onto a subring $\mathscr{R}(\omega)$. Recall that a representation $\pi \in R\left(S_{n}(G)\right)$ lies in $\mathscr{R}(\omega)$ if and only if the restriction $\pi$ to $G^{n}$ is a multiple of $\omega \times \cdots \times \omega$.

With the maps $T_{\omega}$, there is a natural parametrization of the irreducible representations of the wreath product $S_{n}(G)$ by $f \in F_{n}(\hat{G}, y)$. This is done as follows. First, identify $f(\omega)$, for $\omega \in \hat{G}$, with an irreducible representation of the symmetric group $S(|f(\omega)|)$. As in [16], $T_{\omega}(f(\omega))$ is an irreducible representation of $S_{|f(\omega)|}(G)$. Finally, the induction product $\prod\left\{T_{\omega}(f(\omega)): \omega \in \widehat{G}\right\}$ will produce any irreducible of the wreath product $S_{n}(G)$ where $n=|f|$. To simplify notation, we will occasionally identify $f(\omega)$ with its image $T_{\omega}(f(\omega))$ and treat $f$ as the irreducible representation.

In this paper, we will only need the conjugacy classes of $S_{n}(G)$ when $G$ is abelian. They are parametrized by the set $h \in F_{n}(G, Y)$. In outline, this is done as follows. Given a nonnegative integer $m$ and $g \in G$, let $C_{m, g}$ denote the normalized characteristic function of the primitive conjugacy class of $S_{m}(G)$ which consists of all elements $\left(\sigma_{m} ; g_{1}, g_{2}, \ldots, g_{m}\right)$ in $S_{m}(G)\left(\sigma_{m}\right.$ is an $m$-cycle, $\left.g_{1}, \ldots, g_{m} \in G\right)$ such that the cycle product of $g_{1}, \ldots, g_{m}$ is $g$ (see [16] for details). Next, for a Young diagram $Y \in \mathcal{Y}$ and $g \in G$, let $C_{Y, g}=\prod_{j=1}^{r_{*}(Y)} C_{r_{j}(Y), g}$. Finally, for a function $h \in F_{n}(G, Y)$, let $C_{h}=\prod\left\{C_{h(g), g}: g \in G\right\}$. Every conjugacy class has this form.

The algebraic structure for $\mathscr{R}$ extends to the infinite sum of complex-valued class functions $\mathscr{C}=\bigoplus_{n=0}^{\infty} C F\left(S_{n}(G)\right)$, so it forms a graded commutative algebra. Further, each space $C F\left(S_{n}(G)\right)$ admits an inner product from the $L^{2}$-inner product on $S_{n}(G)$ relative to normalized counting measure. Let $\operatorname{Char}(f)$ denote the character of the irreducible $f \in F_{n}(\hat{G}, \mathscr{Y})$ and let $h \in F_{n}(G, \mathscr{Y})$, then $\langle\operatorname{Char}(f), h\rangle$ is the value of the character of the irreducible $f$ at the conjugacy class $h$. Furthermore, the subrings $\mathscr{R}(\omega)$ are mutually orthogonal. Let $\mathscr{C}_{0}$ denote the corresponding algebra for the symmetric groups.

We record two basic formulas.

Branching law. Let $f \in S_{n}(G)$ and $f^{\prime} \in S_{n+1}(G)$. Then $f$ occurs in the restriction $f^{\prime}$ to $S_{n}(G)$ if and only if there exists $\omega_{0} \in \hat{G}$ such that $f(\omega)=f^{\prime}(\omega)$ for all $\omega \neq \omega_{0}$ and $f\left(\omega_{0}\right)$ occurs in the restriction of $f^{\prime}\left(\omega_{0}\right)$ to $S\left(\left|f\left(\omega_{0}\right)\right|\right)$. Further, the representation $f$ occurs in $f^{\prime}$ with multiplicity $\operatorname{dim}\left(\omega_{0}\right)$.

Dimension theorem. Let $f \in S_{n}(G)$, then

$$
\operatorname{dim}(f)=\left(\begin{array}{c}
n \\
n_{1}, \ldots, n_{p}
\end{array}\right) \prod_{j=1}^{p} \operatorname{dim}\left(f\left(\omega_{j}\right)\right) \operatorname{dim}\left(\omega_{j}\right)^{\left|f\left(\omega_{j}\right)\right|}
$$

where $\hat{G}=\left\{\omega_{1}, \ldots, \omega_{p}\right\}, n_{j}=\left|f\left(\omega_{j}\right)\right|$, and $\operatorname{dim}\left(f\left(\omega_{j}\right)\right)$ is the dimension of the corresponding irreducible representation of $S\left(\left|f\left(\omega_{j}\right)\right|\right)$.

The following theorem follows from [4, Section 3].

Theorem 2.1. As a ring, $K_{0}(S(G)) \simeq \mathscr{R} /\left(1-\sum\{\operatorname{dim}(\omega) \omega: \omega \in \hat{G}\}\right)$. 
We now reduce the character theory of infinite wreath products to the abelian case by using the ergodic method of finding characters for locally finite groups. We briefly review this method from the theory of $\mathrm{AF} C^{\star}$-algebras. See [6, Chapter 1] for a general exposition. Because the group $C^{\star}$-algebra of $S(G)$ is an AF-algebra, it possesses an associated dynamical system $(X, \Gamma)$, where $X$ is a certain path space and $\Gamma$ is a locally finite group of path permutations [11]. In particular, $X$ consists of infinite paths through the "multigraph" whose nodes are given by $f \in \widehat{S}_{j}(G), j \geq 0$, such that the two vertices $f$ and $f^{\prime}$ are connected by $k$ edges if $f \in \widehat{S_{j}}(G), f^{\prime} \in \widehat{S_{j+1}}(G)$, and $f \leq f^{\prime}$ where $k$ is the multiplicity of $f$ in $f^{\prime}$. The finite characters are determined by the $\Gamma$-invariant and $\Gamma$-ergodic probability measures $\mu$ on $X$. The measure $\mu$ is determined by its values on the cylinder sets $X_{f}$, where $f \in \widehat{S_{n}}(G)$, for some $n$, and $X_{f}$ consists of all paths that pass through the node $f$. The ergodic method states that the measure $\mu$ is uniquely determined by the limit

$$
\lim _{N \rightarrow \infty} \frac{\# \operatorname{Path}(0, f, G) \# \operatorname{Path}\left(f, f_{N}, G\right)}{\# \operatorname{Path}\left(0, f_{N}, G\right)}=\mu\left(X_{f}\right),
$$

where 0 is the trivial representation of $S_{0}(G)=\{e\}, f_{N} \in \widehat{S_{N}}(G)$, and $\operatorname{Path}\left(f, f^{\prime}, G\right)$ is the set of all finite paths whose initial node is $f$ and whose final node is $f^{\prime}$.

We recognize that \#Path $\left(0, f^{\prime}, G\right)$ is the dimension of the irreducible representation $f^{\prime} \in S_{\left|f^{\prime}\right|}(G)$ which we write as $\operatorname{dim}_{G}\left(f^{\prime}\right)$ or $\operatorname{dim}\left(f^{\prime}\right)$, if the choice of $G$ is clear, while \#Path $\left(f, f^{\prime}, G\right)$ equals the multiplicity of the representation $f$ in the restriction $f^{\prime}$ to $S_{|f|}(G)$ and will be denoted by $\operatorname{dim}_{G}\left(f^{\prime} \backslash f\right)$ or $\operatorname{dim}\left(f^{\prime} \backslash f\right)$, again if the choice of $G$ is clear. For the symmetric group, this multiplicity $\operatorname{dim}\left(Y^{\prime} \backslash Y\right)$, where $Y, Y^{\prime} \in \mathscr{Y}$ and $Y \subset Y^{\prime}$, is also known as the dimension of the skew-diagram formed by the set-difference of the Young diagrams $Y^{\prime}$ and $Y$.

Let $t$ be the associated character of the $C^{\star}(S(G))$. Then the value of $t$ on the minimal projection $e(f)$ is given by

$$
t(e(f))=\lim _{N \rightarrow \infty} \frac{\operatorname{dim}_{G}\left(f_{N} \backslash f\right)}{\operatorname{dim}_{G}\left(f_{N}\right)} .
$$

By the branching law and dimension theorem, we have the relationship

$$
\operatorname{dim}_{G}\left(f^{\prime} \backslash f\right)=\operatorname{dim}_{\mathbb{Z}_{p}}\left(f^{\prime} \backslash f\right) \prod_{\omega \in \hat{G}} \operatorname{dim}(\omega)^{\left|f^{\prime}(\omega)\right|-|f(\omega)|} .
$$

Hence, the ratios of the orders of the path spaces are related as

$$
\frac{\operatorname{dim}_{G}\left(f_{N} \backslash f\right)}{\operatorname{dim}_{G}\left(f_{N}\right)}=\left(\prod_{\omega \in \hat{G}} \frac{1}{(\operatorname{dim} \omega)^{|f(\omega)|}}\right) \frac{\operatorname{dim}_{\mathbb{Z}_{p}}\left(f_{N} \backslash f\right)}{\operatorname{dim}_{\mathbb{Z}_{p}}\left(f_{N}\right)},
$$

where $p=|\widehat{G}|$. Hence, the limit in (2.6) exists for the group $G$ if and only if it exists for $\mathbb{Z}_{p}$.

We sum up this discussion in the following. 
Theorem 2.2. Let $G$ be any finite group. Then the character theory of the infinite wreath product depends solely on the cardinality of $\hat{G}$. If $|G|=\left|\mathbb{Z}_{p}\right|$, identify $\hat{G}$ with $\widehat{\mathbb{Z}}_{p}$, so both $\widehat{S_{n}(G)}$ and $\widehat{S_{n}\left(\mathbb{Z}_{p}\right)}$ can be identified with $F_{n}(X, Y)$ where $|X|=p$. If $f \in F_{n}(X, Y)$, let $c_{f}=$ $\prod\left\{(\operatorname{dim} \omega)^{|f(\omega)|}: \omega \in \hat{G}\right\}$. Then for any finite character $t_{G}$ on $S(G)$,

$$
c_{f} t_{G}\left(e_{G}(f)\right)=t_{\mathbb{Z}_{p}}\left(e_{\mathbb{Z}_{p}}(f)\right) .
$$

It would be interesting to have a version of this result for nonfinite compact groups $G$ but it is not clear what the replacement of $\mathbb{Z}_{p}$ is. For the remainder of the paper, we assume $G$ is a finite abelian group.

\section{Finite characters and primitive ideals}

We give a brief review of the known classification of the finite characters of $S(\infty)$ together with their asymptotic character formula and the ergodic method. Let $x$ denote two nonincreasing sequences $\left\{a_{j}\right\}$ and $\left\{b_{j}\right\}$ of nonnegative reals such that $c=1-\sum\left(a_{j}+\right.$ $\left.b_{j}\right) \geq 0$. We set

$$
F(x, t)=e^{c t} \prod_{j=1}^{\infty} \frac{1+a_{j} t}{1-b_{j} t}=\sum_{n=0}^{\infty} p_{n}(x) t^{n}
$$

where $p_{n}(x)=\sum_{\ell=1}^{\infty}\left(a_{\ell}\right)^{n}-\left(-b_{\ell}\right)^{n}$ are the generalized power sums. For $Y \in \mathcal{Y}$, let $s_{Y}(x)$ denote the corresponding Schur function given by the power series $F(x, t)$, so $s_{Y}(x)=$ $\operatorname{det}\left[p_{r_{i}(Y)-i+j}(x)\right]$. Then the corresponding finite character $t_{x}$ is multiplicative relative to disjoint cycles and is given by $t_{x}(n$-cycle $)=p_{n}(x)$ and $t_{x}(e(Y))=s_{Y}(x)$, where $e(Y)$ is any minimal projection determined by the diagram $Y \in \widehat{S(n)}$. Every finite character has this form [12]. For further discussion of these results, see also $[6,9,13,15]$. We call $x$ the Thoma parameters of the character.

Recall the asymptotics for the $S(\infty)$-characters. Let $Y_{N} \in \mathscr{Y}_{N}$ correspond to an irreducible of $S(N)$ with normalized shifted Frobenius coordinates $a_{N, \ell}=\left(h_{\ell}\left(Y_{N}\right)+1 / 2\right) / N$ and $b_{N, \ell}=\left(v_{\ell}\left(Y_{N}\right)+1 / 2\right) / N$, with $\ell=1,2, \ldots, d_{\#}\left(Y_{N}\right)$. Let $x_{N}$ denote the sequence pair $\left\{a_{N, \ell}, b_{N, \ell}\right\}$. Following [16], let $z_{m}$ denote the normalized characteristic function of $m$ cycles in $S(m)$. Identify as usual $\sigma \in S(m)$ with its image in $S(m+k)$ where its image fixes the complementary indices to $\{1, \ldots, m\}$ among $\{1, \ldots, m+k\}$. Then the character value of $Y_{N}$ at an $m$-cycle $\sigma_{m}$ is given by $\left\langle\operatorname{Char}\left(Y_{N}\right), z_{m} z_{1}^{N-m}\right\rangle$ and

$$
\frac{\left\langle\operatorname{Char}\left(Y_{N}\right), z_{m} z_{1}^{N-m}\right\rangle}{\operatorname{dim}\left(Y_{N}\right)}=\sum_{\ell=1}^{d_{H}\left(Y_{N}\right)}\left(\left(a_{N, \ell}\right)^{m}-\left(-b_{N, \ell}\right)^{m}\right)+O\left(\frac{1}{N}\right),
$$

where $z_{m}$ denotes the normalized characteristic function of the conjugacy class of all $m$ cycles in $S(m)$ and $z_{m} z_{1}^{N-m}$ is the induction product in $\mathscr{C}_{0}$. Then the limit of the normalized sequence of characters exists if and only if $a_{N, \ell} / N \rightarrow a_{\ell}$ and $b_{N, \ell} / N \rightarrow b_{\ell}$ such that $\sum\left(a_{\ell}+b_{\ell}\right)<\infty$. This can be restated relative to the generalized power sums given by the generating function $F_{N}\left(x_{N}, t\right)=\prod_{\ell=1}^{N}\left(1+a_{N, \ell} t\right) /\left(1-b_{N, \ell} t\right)$. 
This framework can be rephrased in terms of the ergodic method. By [6], we have the asymptotic estimate

$$
\frac{\operatorname{dim}\left(Y_{N} \backslash Y_{0}^{*}\right)}{\operatorname{dim}\left(Y_{N}\right)}=s_{Y_{0}^{*}}\left(x_{N}\right)+O\left(\frac{1}{N}\right)
$$

where $s_{Y_{0}^{*}}\left(x_{N}\right)$ is the Schur function relative to $F_{N}\left(x_{N}, t\right)$. Hence, for any finite character $t$ with Thoma parameters $x$, there exists a sequence $\left\{Y_{N}\right\}, Y_{N} \in \widehat{S(N)}$, such that $t\left(e_{Y_{0}^{*}}\right)=$ $s_{Y_{0}^{*}}(x)$ if and only if $r_{\ell}\left(Y_{N}\right) / N \rightarrow a_{\ell}, c_{\ell}\left(Y_{N}\right) \rightarrow b_{\ell}$, and $\sum\left(a_{\ell}+b_{\ell}\right)<\infty$.

By means of the asymptotic character formula and the ergodic method, we will be able to classify all the finite characters of $S(G)$.

Theorem 3.1. The sequence $\left\{\operatorname{Char}\left(f_{N}\right) / \operatorname{dim}\left(f_{N}\right)\right\}_{N=1}^{\infty}$ of normalized irreducible characters $f_{N} \in \widehat{S_{N}(G)}$ has a limit if and only if

$$
\lim _{N \rightarrow \infty} \frac{\left|f_{N}(\omega)\right|}{\left|f_{N}\right|}=q_{\omega}, \quad \sum_{\omega \in \hat{G}} q_{\omega}=1,
$$

and whenever $q_{\omega}>0$, the following limits exist for all $j \geq 1$ :

$$
\lim _{N \rightarrow \infty} \frac{r_{j}\left(f_{N}\right)(\omega)}{\left|f_{N}(\omega)\right|}=a_{j}(\omega), \quad \lim _{N \rightarrow \infty} \frac{c_{j}\left(f_{N}\right)(\omega)}{\left|f_{N}(\omega)\right|}=b_{j}(\omega), \quad \sum_{j=1}^{\infty} a_{j}(\omega)+b_{j}(\omega)<\infty .
$$

Proof. Because of the multiplicative nature of finite characters $[7,13]$, it is enough to establish the limits of the normalized characters on the primitive conjugacy classes which play the role of $n$-cycles in the infinite symmetric group.

Let $f \in F_{N}(\widehat{G}, y)=\widehat{S_{N}}(G)$. We wish to find the character value $\operatorname{Char}(f)$ at the conjugacy class $C_{h}$ which is nothing more than the inner product $\left\langle\operatorname{Char}\left(f_{N}\right), C_{h}\right\rangle$. We will be using the formalism of [16]. By [16, Section 7], we have for a primitive conjugacy class $C_{m, g}$ that

$$
C_{m, g}=\sum\left\{\overline{\omega(g)} T_{\omega}\left(z_{m}\right): \omega \in \hat{G}\right\}
$$

where $g \in G$ and $m$ is a nonnegative integer.

With the usual embedding of $S_{m}(G)$ into $S_{N}(G)$, we analyze the asymptotic behavior of the inner product

$$
\frac{\left\langle\operatorname{Char}\left(f_{N}\right), C_{m, g}\left(C_{1, e}\right)^{N-m}\right\rangle}{\operatorname{dim}\left(f_{N}\right)} .
$$

Let $\widehat{G}=\left\{\omega_{1}, \ldots, \omega_{|G|}\right\}$, so

$$
\left(\sum_{j=1}^{|G|} \overline{\omega(g)} T_{\omega_{j}}\left(z_{m}\right)\right)^{N-m}=\sum\left(\begin{array}{c}
N-m \\
n_{1}, \ldots, n_{|G|}
\end{array}\right) T_{\omega_{1}}^{n_{1}}\left(z_{1}\right) \cdots T_{\omega_{|G|}}^{n_{|G|}}\left(z_{1}\right)
$$

where the sum is over all nonnegative integers $n_{1}, \ldots, n_{|G|}$ that sum to $N-m$. 
By orthogonality, the only nonzero contributions of $C_{m, g}\left(C_{1, e}\right)^{N-m}$ in the inner product

$$
\left\langle\operatorname{Char}\left(f_{N}\right), C_{m, g}\left(C_{1, e}\right)^{N-m}\right\rangle=\left\langle\operatorname{Char}\left(f_{N}\left(\omega_{1}\right)\right) \cdots \operatorname{Char}\left(f_{N}\left(\omega_{|G|}\right)\right), C_{m, g}\left(C_{1, e}\right)^{N-m}\right\rangle
$$

occur when for some $j_{0}$, we have $m+n_{j_{0}}=\left|f\left(\omega_{j_{0}}\right)\right|$ and for $j \neq j_{o}, n_{j}=\left|f\left(\omega_{j}\right)\right|$. Again we emphasize that the products here are induction products in $\mathscr{b}$.

For convenience, set $n_{i}=\left|f\left(\omega_{i}\right)\right|$, and let $A(N-m, j)$ denote the multinomial coefficient $\left(\begin{array}{c}N-m \\ m_{1}, \ldots, m_{|G|}\end{array}\right)$ where $m_{i}=n_{i}$, for $i \neq j$, and $m_{j}=n_{j}-m$. Further, set $M_{j}$ to be the monomial product in $\mathscr{C}$ :

$$
M_{j}=T_{\omega_{1}}\left(z_{1}\right)^{n_{1}} \cdots T_{\omega_{j-1}}\left(z_{1}\right)^{n_{j-1}} T_{\omega_{j}}\left(z_{1}\right)^{n_{j}-m} T_{\omega_{j+1}}\left(z_{1}\right)^{n_{j+1}} \cdots T_{\omega_{|G|}}\left(z_{1}\right)^{n_{|G|}} .
$$

We also need the elementary asymptotic estimate for multinomial coefficients where $p, n_{0}, k_{1}, \ldots, k_{p}$ will be treated as fixed:

$$
\frac{\left(\begin{array}{c}
N-n_{0} \\
K_{1}-k_{1}, \ldots, K_{p}-k_{p}
\end{array}\right)}{\left(\begin{array}{c}
N \\
K_{1}, \ldots, K_{p}
\end{array}\right)}=\left(\frac{K_{1}}{N}\right)^{k_{1}} \cdots\left(\frac{K_{p}}{N}\right)^{k_{p}}+O\left(\frac{1}{N}\right)
$$

Hence, the ratio $\left(\begin{array}{c}N-n_{0} \\ K_{1}-k_{1}, \ldots, K_{p}-k_{p}\end{array}\right) /\left(\begin{array}{c}N \\ K_{1}, \ldots, K_{p}\end{array}\right)$ has a limit if and only if for each $j=1, \ldots, p$, the sequence $\left\{K_{j} / N\right\}$ converges with limit $q_{j}$; hence the limit of the quotient is $\prod q_{j}^{k_{j}}$.

We now have the simplifications

$$
\begin{aligned}
\langle\operatorname{Char} & \left.\left(f_{N}\right), C_{m, g}\left(C_{1, e}\right)^{N-m}\right\rangle=\sum_{j=1}^{|G|} \overline{\omega_{j}(g)} A(N-m, j)\left\langle\operatorname{Char}\left(f_{N}\right), T_{\omega_{j}}\left(z_{m}\right) M_{j}\right\rangle \\
= & \sum_{j=1}^{|G|} \overline{\omega_{j}(g)} A(N-m, j)\left\langle\operatorname{Char}\left(f_{N}\left(\omega_{j}\right)\right), T_{\omega_{j}}\left(z_{m}\right) T_{\omega_{j}}\left(z_{1}\right)^{\left|f_{N}\left(\omega_{j}\right)\right|-m}\right\rangle \\
& \times \prod_{i \neq j}\left\langle\operatorname{Char}\left(f_{N}\left(\omega_{i}\right)\right), z_{1}^{\left|f_{N}\left(\omega_{i}\right)\right|}\right\rangle \\
= & \sum_{j=1}^{|G|} \overline{\omega_{j}(g)} A(N-m, j)\left\langle\operatorname{Char}\left(f_{N}\left(\omega_{j}\right)\right), z_{m} z_{1}^{\left|f_{N}\left(\omega_{j}\right)\right|-m}\right\rangle \prod_{i \neq j} \operatorname{dim}\left(f_{N}\left(\omega_{i}\right)\right),
\end{aligned}
$$

where we used the fact that $\left\langle\operatorname{Char}\left(f_{N}\left(\omega_{i}\right)\right), z_{m}^{|f(\omega)|}\right\rangle$ is the value of the character of the irreducible representation $f_{N}\left(\omega_{i}\right)$ of $S\left(\left|f_{N}\left(\omega_{i}\right)\right|\right)$ at the identity which reduces to the dimension of the representation. 
1372 Character theory of infinite wreath products

We conclude that the normalized character value is

$$
\begin{aligned}
& \frac{\left\langle\operatorname{Char}\left(f_{N}\right), C_{m, g}\left(C_{1, e}\right)^{N-m}\right\rangle}{\operatorname{dim}\left(f_{N}\right)} \\
& \quad=\sum_{j=1}^{|G|} \overline{\omega_{j}(g)} \frac{A(N-m, j)}{\operatorname{dim}\left(f_{N}\right)}\left\langle\operatorname{Char}\left(f_{N}\left(\omega_{j}\right)\right), z_{m} z_{1}^{\left|f_{N}\left(\omega_{j}\right)\right|-m}\right\rangle \prod_{i \neq j} \operatorname{dim}\left(f_{N}\left(\omega_{i}\right)\right) .
\end{aligned}
$$

Since the character value on $S(N)$, $\left\langle\operatorname{Char}\left(f_{N}\left(\omega_{j}\right)\right), z_{m} z_{1}^{\left|f_{N}\left(\omega_{j}\right)\right|-m}\right\rangle$ can be handled with the asymptotic character formula for $S(\infty)$, we will give now a full asymptotic expansion for the normalized character value:

$$
\begin{aligned}
\frac{\left\langle\operatorname{Char}\left(f_{N}\right), C_{m, g}\left(C_{1, e}\right)^{N-m}\right\rangle}{\operatorname{dim}\left(f_{N}\right)}= & \sum_{i=1}^{|G|} \frac{\omega_{i}(g)}{(}\left[\left(\frac{\left|f_{N}\left(\omega_{i}\right)\right|}{N}\right)^{m}+O\left(\frac{1}{N}\right)\right] \\
& \times\left(\sum_{\ell=1}^{d_{\#}\left(f_{N}\left(\omega_{i}\right)\right)} a_{\ell}\left(f\left(\omega_{i}\right)\right)^{m}-b_{\ell}\left(f\left(\omega_{i}\right)\right)^{m}+O\left(\frac{1}{\left|f_{N}\left(\omega_{i}\right)\right|}\right)\right),
\end{aligned}
$$

where $a_{\ell}(\omega)=\left(h_{\ell}\left(f_{N}(\omega)\right)+1 / 2\right) /\left|f_{N}(\omega)\right|$ and $b_{\ell}(\omega)=\left(v_{\ell}\left(f_{N}(\omega)\right)+1 / 2\right) /\left|f_{N}(\omega)\right|$ are the normalized shifted Frobenius coordinates.

If $\left|f_{N}\left(\omega_{i}\right)\right| / N \rightarrow 0$, then this term has no contribution to the sum. So, by passing to a subsequence, if necessary, assume $\left|f_{N}\left(\omega_{i}\right)\right| / N \rightarrow q_{\omega}>0$. By [13, Lemma 2], (3.13) possesses a limit for such $\omega$ if and only if both $a_{\ell}(f(\omega))$ and $b_{\ell}(f(\omega))$ possess limits, say $a_{\ell}(\omega)$ and $b_{\ell}(\omega)$, respectively, for all $\ell$, together with the normalization $\sum_{\ell} a_{\ell}(\omega)+b_{\ell}(\omega)=$ $1-c(\omega) \geq 0$. In particular, this term has contribution $q_{\omega} p_{m}(z(\omega))$ to the sum. Since the characters $\omega$ are a basis for functions over $G$, we find that distinct limits give distinct finite characters.

THEOREM 3.2. The finite characters $t$ of $S(G)$ are parametrized by $\left(q_{\omega}, x(\omega): \omega \in \hat{G}\right)$ where $q_{\omega} \geq 0$ with $\sum q_{\omega}=1$ and $x(\omega)=\left(a_{\ell}(\omega), b_{\ell}(\omega)\right)$ are Thoma parameters for each $\omega$. On the primitive conjugacy class with $g \in G$ and $m$ a nonnegative integer, the value of $t$ is

$$
t\left(C_{m, g}\right)=\sum\left\{\overline{\omega(g)} \sum_{\ell=1}^{\infty}\left(q_{\omega} a_{\ell}(\omega)\right)^{m}-\left(q_{\omega} b_{\ell}(\omega)\right)^{m}: \omega \in \widehat{G}\right\},
$$

and, on the minimal projection $e(f)$ with $f \in F_{n}(\hat{G}, y)$, is

$$
\prod\left\{q_{\omega}^{|f(\omega)|} s_{f(\omega)}(x(\omega)): \omega \in \hat{G}\right\} .
$$

In particular, $q_{\omega}=t(e(\omega))$ where $e(\omega)$ is the minimal projection corresponding to $\omega \in \widehat{S_{1}(G)}$ in $C^{\star}\left(S_{1}(G)\right)$.

Proof. The evaluation of the finite character $t$ on a primitive conjugacy class follows from the proof of the previous theorem. 
To obtain the values of the finite character $t$ on minimal projections in $C^{\star}(G)$, we use the ergodic method. For $f_{0}^{*} \in \widehat{S_{n}(G)}$, we find

$$
\operatorname{dim}\left(f_{N} \backslash f_{0}^{*}\right)=\left(\begin{array}{c}
\left|f_{N}\right|-\left|f_{0}^{*}\right| \\
\left|f_{0}^{*}\left(\omega_{1}\right)\right|, \ldots,\left|f_{0}^{*}\left(\omega_{|G|}\right)\right|
\end{array}\right) \prod_{j=1}^{|G|} \operatorname{dim}\left(f_{N}\left(\omega_{j}\right) \backslash f_{0}^{*}\left(\omega_{j}\right)\right) .
$$

Hence, the value of finite character $t$ at the minimal projection $e\left(f_{0}^{*}\right)$ is given by the limit:

$$
\begin{aligned}
& t\left(e\left(f_{0}^{*}\right)\right)=\lim _{N \rightarrow \infty} \frac{\operatorname{dim}\left(f_{N} \backslash f_{0}^{*}\right)}{\operatorname{dim}\left(f_{N}\right)} \\
& =\lim _{N \rightarrow \infty} \frac{\left(\begin{array}{c}
\left|f_{N}\right|-\left|f_{0}^{*}\right| \\
\left|f_{0}^{*}\left(\omega_{1}\right)\right|, \ldots,\left|f_{0}^{*}\left(\omega_{|G|}\right)\right|
\end{array}\right) \prod_{j=1}^{|G|} \operatorname{dim}\left(f_{N}\left(\omega_{j}\right) \backslash f_{0}^{*}\left(\omega_{j}\right)\right)}{\left.\left(\left|f_{N}\left(\omega_{1}\right)\right|, \ldots,\left|f_{N}\right| f_{|G|}\right) \mid\right) \prod_{j=1}^{|G|} \operatorname{dim}\left(f_{N}\left(\omega_{j}\right)\right)} .
\end{aligned}
$$

Their limits are

$$
\prod_{\omega \in \hat{G}} q_{\omega}^{|f(\omega)|} e_{f_{0}^{*}}(x)
$$

In particular,

$$
\lim _{N \rightarrow \infty} \frac{\left|f_{N}\left(\omega_{i}\right)\right|}{\left|f_{N}\right|}=t\left(e\left(f_{1, i}\right)\right)=t\left(e\left(\omega_{i}\right)\right)
$$

where $f_{0}^{*}=f_{1, i} \in S_{1}(G)=G$ such that $\left|f_{1, i}\left(\omega_{i}\right)\right|=1$ and $\left|f_{1, i}\left(\omega_{j}\right)\right|=0$ for $j \neq i$. Hence, $q_{\omega}$ has the desired interpretation.

We will call the parameters $\left(q_{\omega}, x(\omega): \omega \in \widehat{G}\right)$ from Theorem 3.2 for a finite character $t$ of $S(G)$ its invariant parameters.

The method in [13] or [3] can be used to classify the primitive ideals of $C^{\star}(S(G))$ by means of the branching law. For each $\omega \in \widehat{G}$, we let $Y(\omega)$ be an infinite Young diagram described in Section 1, so $\mathscr{I}_{k(\omega), \ell(\omega)}+Y_{0}(\omega)$, where $k(\omega)$ is the number of infinite rows of $Y(\omega)$ and $\ell(\omega)$ is the number of infinite columns of $Y(\omega)$. Note: we allow either $k(\omega)$ or $\ell(\omega)$ to be $\infty$. In either case, we set $Y_{0}(\omega)=\varnothing$.

A primitive ideal $J$ is determined by the $\hat{G}$-tuple:

$$
\left(k(\omega), \ell(\omega), Y_{0}(\omega): \omega \in \hat{G}\right)=\left(k, \ell, Y_{0}\right) .
$$

Hence, the minimal projection $e(f) \in J\left(k, \ell, Y_{0}\right)$, where $f \in \widehat{S_{n}}(G)$, if and only if $f(\omega)$ is not a subset of $\mathscr{I}_{k(\omega), \ell(\omega)}+Y_{0}(\omega)$, for some $\omega \in \hat{G}$.

The multiplicative nature of finite characters together with the determination of the kernels of finite characters of $S(\infty)$ in [7] give the kernels of the finite characters of 
infinite wreath products. Suppose $t$ has invariant parameters $x=\left\{a_{j}(\omega), b_{j}(\omega): \omega \in \hat{G}\right\}$ and $\left\{q_{\omega}: \omega \in \hat{G}\right\}$. If $q_{\omega}=0$, then $k(\omega)=\ell(\omega)=0$, so $\mathscr{I}_{k(\omega), \ell(\omega)}=\left(\mathbb{Z}^{2}\right)^{+}$. If $q_{\omega}>0$ and $c(\omega)>0$, then $k(\omega)=\ell(\omega)=\infty$. If $q_{\omega}>0$ and $c(\omega)=0$, let $k(\omega)$, respectively, $\ell(\omega)$ be the largest index such that $a_{j}(\omega)>0$, respectively, $b_{j}(\omega)>0$. Then the kernel (or the primitive ideal) determined by $t$ is $\{(k(\omega), \ell(\omega), \varnothing): \omega \in \hat{G}\}$. An immediate consequence of this calculation is the following.

THeOREm 3.3. A primitive ideal $J=J\left(k, \ell, Y_{0}\right)$ is the kernel of a finite character if and only if $Y_{0}=\varnothing$, for all $\omega \in \hat{G}$.

We close the section with two observations.

(1) The unitary (1-dimensional) characters of $S(G)$ are given by $\Omega_{0,+}\left(\sigma ; g_{1}, \ldots, g_{n}\right)=$ $\omega_{0}\left(g_{1} \cdots g_{n}\right)$ or $\Omega_{0,-}\left(\sigma ; g_{1}, \ldots, g_{n}\right)=\operatorname{sgn}(\sigma) \omega_{0}\left(g_{1} \cdots g_{n}\right)$ where $\omega_{0} \in \hat{G}$. The invariant parameters for $\Omega_{0,+}$, respectively, $\Omega_{0,-}$, are $q_{\omega}=0$ for $\omega \neq \omega_{0} ; q_{\omega_{0}}=1$ and $a_{1}\left(\omega_{0}\right)=1$, respectively, $b_{1}\left(\omega_{0}\right)=1$.

(2) The parameters for the finite characters of $S(G)$ that come from the quotient group $S(G) / G_{\infty} \simeq S(\infty)$ where $G_{\infty}$ is the normal subgroup $\bigcup_{n=1}^{\infty} G^{n}$ are determined by $q_{\omega_{1}}=1$ where $\omega_{1}$ is the constant character 1 on $G$.

\section{Infinite characters}

The aim of this section is to classify the infinite characters of $S(G)$ using Riesz ring techniques. We recall some concepts from Riesz group and ring theory. First, an ordered group $G$ is a Riesz group provided given any elements $a_{i}, b_{j} \in G, i, j=1,2$, there exists an element $c \in G$ with $a_{i} \leq c \leq b_{j}$ for any choice $i, j=1,2$. For any unital AF-algebra, its $K_{0}$-group is a Riesz group. A Riesz group is a Riesz ring provided it has a multiplication that is consistent with the order structure.

If $J$ is the kernel of a character $t$, whether finite or infinite, then $t$ corresponds to a faithful character of the primitive quotient $A=C^{\star}(S(G)) / J$. Further, with the induction product described in Section $1, K_{0}(A)$ is a commutative ring. In particular, $K_{0}(A)$ for a primitive quotient $A$ is a Riesz ring.

Let $H$ denote a Riesz group with nonzero positive elements $a$ and $b$. We say that $a$ is infinitely small relative to $b$ if $n a \leq b$ for all $n \in \mathbb{Z}^{+}$. If $a$ is infinitely small, then $a$ must lie in the kernel of every state of $H$. In particular, if $H$ is a unital Riesz ring with a nonzero infinitely small element, then $H$ can have no faithful finite extremal states.

We need the following theorem found in Antony Wassermann's unpublished University of Pennsylvania Ph.D. thesis. Although the thesis was widely circulated, it was never published. A version of this result with a proof by Wassermann appears in an appendix to [3].

Theorem 4.1. If $R$ is a Riesz ring with no positive zero divisors, then $R$ has no faithful infinite characters.

It is routine to check that the primitive quotients by the ideals $J=J\left(k, \ell, Y_{0}\right)$ where $Y_{0}(\omega)=\varnothing$, for all $\omega \in \hat{G}$, have no positive zero divisors. In another study [4], we found for several inductive limit groups that a primitive ideal $J$ is integral (i.e., the $K_{0}$-ring of 
the primitive quotient is a commutative ring with no positive zero divisors) if and only if $J$ is the kernel of a finite factor representation.

Throughout this section, we let $J=J\left(k, \ell, Y_{0}\right)$ where for some $\omega_{0} \in \hat{G}, Y_{0}\left(\omega_{0}\right) \neq \varnothing$ where we use the notation introduced in (1.3). Recall that $k$ denotes the number of infinite rows and $\ell$ the number of infinite columns, so the shape of such an infinite Young diagram is an infinite "L." The finite diagram $Y_{0}$ denotes the remaining contribution to the shape. We will write $f \subset Y+I(k, \ell)$ if $f(\omega) \subset Y(\omega)+I(k(\omega), \ell(\omega))$ for all $\omega$. We use the same convention for other standard set operations.

We call a primitive ideal integral if the condition that $a, b \in K_{0}(S(G) / I)$ are nonzero and positive implies that their product $a b$ is nonzero. We let $J_{a}$ denote the smallest integral primitive ideal that contains $J$, so that $J_{a}=J(k, \ell)$ (where we omit $Y_{0}$ here since they are all equal to $\varnothing)$.

Proposition 4.2. The primitive quotient $A=C^{\star}(S(G)) / J$ contains a nonzero ideal $B$ which is stably isomorphic to an ideal $B_{a}$ of $A_{a}=C^{\star}(S(G)) / J_{a}$.

Proof. Consider the projections $e(f)$, where $f \in \widehat{S_{n}}(G)$, in $A$ satisfy the condition

$$
f \backslash I(k, \ell)=Y_{0}+(k, \ell) .
$$

We associate to $f(\omega)$, the Young diagram $f_{a}(\omega)=f(\omega) \backslash\left(Y_{0}(\omega)+(k(\omega), \ell(\omega))\right)$. Next consider $e\left(f_{a}\right)$ as a projection in $A_{a}$. By the branching law, the ideal $B$ in $A$ generated by all projections $e(f)$ satisfying (4.1) is stably isomorphic to the ideal $B_{a}$ in $A_{a}$ generated by the projections $e\left(f^{0}\right)$, where $f^{0} \supset\{(i, j): i \leq k(\omega)$ and $j \leq \ell(\omega)\}$, which are rectangular diagrams.

We need to strengthen Proposition 4.2 in order to show that $B$ is the norm closure of the ideal of definition of any faithful character of $A$. We study this question in terms of multiplication of $K_{0}(A)$.

Proposition 4.3. Let $J=J\left(k, \ell, Y_{0}\right)$ with $\left|Y_{0}\right| \geq 1$ with primitive quotient $A=C^{\star}(S(G)) / J$. If $f_{1} \backslash I(k, \ell)=Y_{0}+(k, \ell)$, then the following identities hold in $K_{0}(A)$ :

(1) if $f_{2} \not \subset I(k, \ell)$, then $e\left(f_{1}\right) \circ e\left(f_{2}\right)=0$;

(2) if $f_{2} \in \mathscr{I}(k, \ell)$, then $e\left(f_{1}\right) \circ e\left(f_{2}\right) \neq 0$.

Proof. For the first identity, it suffices to show that $e(f) \circ e(f)=0$, where $f$ is chosen, so $f(\omega)$ is the smallest diagram that contains $Y_{0}(\omega)+(k(\omega), \ell(\omega))$. Without loss of generality, we may assume $G=\{e\}$; that is, we check only the $S(\infty)$ case.

We set $k^{\prime}=r_{1}(f)>k$ and $\ell^{\prime}=c_{1}(f)>1$. Let $p$ be a term in the decomposition of $f \circ f$, which is described by the Littlewood-Richardson rule [8], such that $p \subset J\left(k, \ell, Y_{0}\right)$. According to the Littlewood-Richardson rule, the terms $p$ are determined by the proper insertion of integers into the Young diagram. Let $a_{i}$ be the number of $i$ 's placed in the $i$ th row of $p$. By hypothesis, $a_{1} \geq 1$; moreover, each $a_{i} \geq 1,1 \leq i \leq \ell$, since $r_{j}(p) \leq k, j>\ell^{\prime}$. Thus, $(\ell+1)$ entries must be placed in the first $k$ distinct columns of $p$. This is impossible, so $e(f) \circ e(f)=0$ in $K_{0}(A)$. The second identity follows by the same method by applying the Littlewood-Richardson rule.

We record the lemma from [4, Section 5]. 
Lemma 4.4. Let $R$ be a commutative unital Riesz ring, with identity e. Suppose $S$ is an ideal of $R$ with order unit $f$, with $f \leq e$. If $S$ has trivial multiplication, then for any $a \in R^{+} \backslash S^{+}$ with $a \circ f \neq 0$,

$$
a=a \circ(e-f)^{n}+n a \circ f, \quad n \geq 0,
$$

where $a \circ(e-f)^{n} \neq 0$. In particular, $f$ is infinitely small relative to $e$.

Theorem 4.5. Let $J=J\left(k, \ell, Y_{0}\right)$ be a primitive ideal with $\left|Y_{0}\right| \geq 1$. Then the primitive quotient $A=C^{\star}(S(G)) / J$ contains an ideal $B$ such that

(1) the faithful characters of $A$ are all infinite and are in natural bijection with the faithful finite characters of $C^{\star}(S(G)) / J_{a}$;

(2) the norm closure of the ideal of definition of any faithful character of $A$ is exactly $B$.

Proof. (1) Recall that there is a natural bijection between the faithful characters of any separable primitive $C^{\star}$-algebra $\mathscr{A}$ with any closed nonzero ideal $\mathscr{S}$ where the map from $\mathscr{A}$ to $\mathscr{F}$ is given by restriction while the extension $T$ of a faithful character $\tau$ of $\mathscr{F}$ to $\mathscr{A}$ is given by

$$
T(x)=\sup \{\tau(b): 0 \leq b \leq x, b \in B\},
$$

where $x \in \mathscr{A}^{+}$(see [7, page 28]). Hence, there is a natural bijection between the faithful characters of $A$ and $A_{a}$, where $A_{a}$ is given in Proposition 4.2. Furthermore, $A_{a}$ has no faithful infinite characters by Theorem 4.1, so all the faithful characters of $A$ are classified by the finite characters of $A_{a}$.

(2) By Proposition 4.3, $K_{0}(A)$ has a nontrivial multiplication while the multiplication restricted to $K_{0}(B)$ is trivial. By Lemma 4.4, any faithful character $T$ of $A$ must be infinite; in fact, $T(e(f))=+\infty$ for any $f \subset I(k, \ell)$, while $T(e(f))<\infty$ for $f \backslash I(k, \ell)=Y_{0}+(k, \ell)$. We conclude that the norm closure of domain of $T$ must contain the ideal $B$. To show equality requires further calculation.

Let $Y_{*}$ denote a diagram obtained from $Y_{0}$ by deleting exactly one node. Next, for a diagram $Y_{1}$, we introduce the notation $\operatorname{Rep}\left(n, Y_{1}\right)$ for the set of all $f \in \widehat{S_{n}(G)}$ for some $n$ such that $f \backslash I(k, \ell)=Y_{1}+(k, \ell)$. So, for $f \in \operatorname{Rep}\left(n, Y_{0}\right), e(f) \in B$. We will also identify representations with their images in $K_{0}(A)$. As a consequence, if $\pi_{1}$ is a representation of $S_{m}(G)$ and $\pi_{2}$ of $S_{n}(G)$, then their induction product $\pi_{1} \circ \pi_{2}=\operatorname{Ind}_{S_{m}(G) \times S_{n}(G)}^{S_{m+n}(G)}\left(\pi_{1} \times \pi_{2}\right)$ reduces to the image in $K_{0}(A)$ of the subrepresentation of their induction product whose irreducible components $f$ satisfy $f \backslash I(k, \ell) \subset Y_{0}$. Let $1_{A}$ denote the multiplicative identity for $K_{0}(A)$ which is equivalent to the induction product with respect to the left regular representation of $S_{1}(G)=G$.

Given any representation $\pi$ of $S_{m}(G)$, let $\operatorname{sub}\left(\pi, Y_{*}\right)$ denote the subrepresentation of $\pi$ whose irreducible components $f$ satisfy $f \backslash I(k, \ell)=Y_{*}$. Then for $f_{*} \in \operatorname{Rep}\left(n, Y_{1}\right)$, let $f_{*, 1}=\operatorname{sub}\left(f_{*}, Y_{*}\right)$ and $f_{0}=\operatorname{sub}\left(f_{*}, Y_{0}\right)$. By the branching rule, we find that $\operatorname{sub}\left(f_{*, 1} \circ\right.$ $\left.1_{A}, Y_{0}\right)$ is equivalent to $f_{0} \circ 1_{A}$. In particular, in $K_{0}(A)$, working with minimal projections, we have $e\left(f_{*}\right) \geq 2 e\left(f_{0}\right)$. We obtain $e\left(f_{*}\right) \geq k e\left(f_{0}\right)$ in $K_{0}(A)$ for all $k$, by iterating this construction. We conclude that $T\left(e\left(f_{*}\right)\right)=\infty$. 
Since $A$ is a primitive $C^{\star}$-algebra, any $e(f) \in \operatorname{Rep}\left(Y_{2}\right)$, where $Y_{2}$ is nonempty, must contain a subprojection of the form $e\left(f_{*}\right)$, where $f_{*} \in \operatorname{Rep}\left(Y_{*}\right)$, for some $Y_{*}$. Hence, the norm closure of the domain of $T$ is exactly $B$.

COROLlary 4.6. The natural map from the space of quasiequivalence classes of traceable factor representations of $S(G)$ to $\operatorname{Prim}(S(G))$ is surjective.

Corollary 4.7. Let $T$ be an infinite character on $C^{\star}(S(G))$ with kernel $J\left(k, \ell, Y_{0}\right)$ and $\left|Y_{0}\right| \geq 1$. Then there is a finite character $T_{\text {fin }}$ with kernel $J(k, \ell)$ such that

$$
T(e(f))= \begin{cases}T_{\mathrm{fin}}\left(e\left(f^{\prime}\right)\right), & \text { if } f \backslash \Phi(k, \ell)=Y_{0}+(k, \ell), \\ +\infty, & \text { if } f \backslash \Phi(k, \ell) \text { is a strict subset of } Y_{0}+(k, \ell), \\ 0, & \text { otherwise, }\end{cases}
$$

where $f^{\prime}=f \backslash\left(Y_{0}+(k, \ell)\right)$ and $f \in \widehat{S_{N}(G)}$.

The order structure on $K_{0}(S(G))$ can be determined very analogously as for $S(\infty)$ (see [7] for the infinite symmetric group case). Recall that both finite and infinite characters are needed to determine the positivity of a nonzero element $x$ in a Riesz group: $x$ is positive if and only if $x$ is contained in a minimal order ideal $I$ such that (i) for all finite character $t$ whose restriction to $I$ is nonzero, $t(x)>0$, and (ii) for all infinite characters $T$ whose restriction to $I$ is finite and nonzero, $T(x)>0$.

Following [15], we let $x=\sum\left\{c_{f} e(f): f \in \widehat{S_{N}(G)}, c_{f} \in \mathbb{Z}\right\}$ be a nonzero element from $K_{0}(S(G))$. For each $\omega \in \hat{G}$, let $\operatorname{core}(x)=\bigcap\left\{f: c_{f} \neq 0\right\}$; that is, $\operatorname{core}(x)(\omega)=\bigcap\{f(\omega)$ : $\left.c_{f} \neq 0\right\}$, for each $\omega \in \hat{G}$. Let $\operatorname{supp}(x(\omega))=\left\{f(\omega): c_{f} \neq 0\right\}$. Choose $\left(k_{1}, \ell_{1}\right)$, so $S\left(k_{1}, \ell_{1}\right)$ the set of all $\omega \in \hat{G}$ such that $\operatorname{core}(x)(\omega) \not \subset I(k(\omega), \ell(\omega))$ is nonempty. For $\omega_{0} \in S$, choose $Y_{\min }\left(\omega_{0}\right)$ to be minimal relative to inclusion among all solutions of the set identity

$$
Y^{*}+\left(k_{1}\left(\omega_{0}\right), \ell\left(\omega_{0}\right)\right)=Y \backslash I\left(k_{1}(\omega), \ell_{1}(\omega)\right),
$$

where $Y$ is from $\operatorname{supp}\left(x\left(\omega_{0}\right)\right)$. Let $\mathscr{Y}\left(\omega_{0}\right)$ denote the set of all such diagrams $Y_{\min }\left(\omega_{0}\right)$.

Theorem 4.8. A nonzero element $z=\sum\left\{c_{f} e(f): f \in \widehat{S_{n}}(G)\right\}$ of $K_{0}(S(G))$ is positive if and only if the following hold.

(1) If core $(x) \subset I(k, \ell)$ and $t$ is a finite character whose kernel is $J(k, \ell)$, then $t(x)>0$.

(2) Choose $\left(k_{1}, \ell_{1}\right)$ so that $S\left(k_{1}, \ell_{1}\right)$ is nonempty. Choose $Y_{1}$, so either $Y_{1}(\omega)=\varnothing$ or, for at least one choice of $\omega$, as $Y_{1}(\omega)$ is from $\mathscr{Y}(\omega)$. If T is an infinite character with kernel $J\left(k, \ell, Y_{1}\right)$, then $T(x)>0$.

We conclude with several comments and examples.

(1) Let $J=J\left(k, \ell, Y_{0}\right) \in \operatorname{Prim}(S(G))$ such that for each $\omega \in \hat{G}, k(\omega)+\ell(\omega)=1$, and $Y_{0}(\omega)=\varnothing$ with primitive quotient $A$. Then the Bratteli diagram of $A$ is a multidimensional version of the Pascal triangle. For the invariant parameters for these characters, we find $a_{1}(\omega)=k(\omega)$ and $b_{1}(\omega)=\ell(\omega)$ and no constraint for $q_{\omega}$. See [7] for a discussion of its $K$-theory. It is also routine to describe its general factor representation of $A$ from the results in [1]. 
(2) A primitive ideal $J=J\left(k, \ell, Y_{0}\right)$ of $C^{\star}(S(G))$ is type $\mathrm{I}_{\infty}$ if and only if there exists $\omega_{0} \in \hat{G}$ such that (1) for all $\omega \neq \omega_{0}, k(\omega)=\ell(\omega)=\infty$, (2) for $\omega_{0}, k\left(\omega_{0}\right)+\ell\left(\omega_{0}\right)=$ 1 (i.e., there is a single infinite row or column), and (3) $Y_{0}\left(\omega_{0}\right) \neq \varnothing$. Note if $Y_{0}\left(\omega_{0}\right)=\varnothing$, the ideal $J$ is type I but finite.

(3) The primitive ideal space of a locally finite group $H$ cannot always be parametrized by the kernels of traceable factor representations unlike the examples in this paper and for connected Lie groups. Using the results of [2], this holds even if $K_{0}(H)$ admits a nontrivial ring structure. Let $H$ denote the weak infinite product of $S(\infty)$, so $C^{\star}(H)$ is the infinite tensor product of the single $C^{\star}(S(\infty))$. In the notation of [2], let $J$ be the primitive ideal of $C^{\star}(H)$ such that $J=\left(J_{i}\right)$ where each $J_{i}$ is the kernel of the same traceable representation irreducible infinitedimensional representation of $C^{\star}(S(\infty))$. It is easy to see that such representations exist. By [2, Theorem 6.5], $J$ cannot be the kernel of any traceable factor representation.

(4) In [14], it is commented that in the product space realization of the finite factor representations of $S(\infty)$, the representation automatically becomes a type III factor representation when the product measure no longer has constant factors. This is not true in general. For $S(\infty)$, certain factor representations with primitive kernels consisting of two infinite rows or an infinite row and column provide examples. More general cases are combinatorially more difficult [1].

\section{References}

[1] B. M. Baker and R. T. Powers, Product states of certain group-invariant AF-algebras, J. Operator Theory 16 (1986), no. 1, 3-50.

[2] B. E. Blackadar, Infinite tensor products of $C^{*}$-algebras, Pacific J. Math. 72 (1977), no. 2, 313334.

[3] R. Boyer, Infinite traces of AF-algebras and characters of $U(\infty)$, J. Operator Theory 9 (1983), no. 2, 205-236.

[4] Characters of the infinite symplectic group-a Riesz ring approach, J. Funct. Anal. 70 (1987), no. 2, 357-387.

[5] J. Faraut, Infinite Dimensional Harmonic Analysis and Probability, CIMPA, Nice, France, and Tata Institute of Fundamental Research, Mumbai, 2002.

[6] S. V. Kerov, Asymptotic Representation Theory of the Symmetric Group and Its Applications in Analysis, Translated from the Russian manuscript by N. V. Tsilevich. With a foreword by A. Vershik and comments by G. Olshanski. Translations of Mathematical Monographs, vol. 219, American Mathematical Society, Rhode Island, 2003.

[7] S. V. Kerov and A. M. Vershik, Characters, factor representations and K-functor of the infinite symmetric group, Operator Algebras and Group Representations, Vol. II (Neptun, 1980), Monogr. Stud. Math., Pitman, Massachusetts, 1984, pp. 23-32.

[8] I. G. Macdonald, Symmetric Functions and Hall Polynomials, 2nd ed., Oxford Mathematical Monographs, The Clarendon Press, Oxford University Press, New York, 1995.

[9] A. Okounkov, Thoma's theorem and representations of the infinite bisymmetric group, Funct. Anal. Appl. 28 (1994), no. 2, 101-107.

[10] Y. Roichman, Upper bound on the characters of the symmetric groups, Invent. Math. 125 (1996), no. 3, 451-485.

[11] Ş. Stratila and D. Voiculescu, Representations of AF-Algebras and of the Group $U(\infty)$, Lecture Notes in Math., vol. 486, Springer, New York, 1975. 
[12] E. Thoma, Die unzerlegbaren, positiv-definiten Klassenfunktionen der abzählbar unendlichen, symmetrischen Gruppe, Math. Z. 85 (1964), 40-61 (German).

[13] A. M. Vershik and S. V. Kerov, Asymptotic theory of characters of the symmetric group, Funct. Anal. Appl. 15 (1981), no. 4, 246-255.

[14] Locally semisimple algebras, combinatorial theory and the $K_{0}$-functor, J. Soviet Math. 38 (1987), 1701-1733.

[15] - The Grothendieck group of infinite symmetric group and symmetric functions (with the elements of the theory of $K_{0}$-functor of AF-algebras), Representation of Lie Groups and Related Topics (A. M. Vershik and D. P. Zhelobenko, eds.), Adv. Stud. Contemp. Math., vol. 7, Gordon and Breach, New York, 1990, pp. 39-117.

[16] A. V. Zelevinsky, Representations of Finite Classical Groups, Lecture Notes in Mathematics, vol. 869, Springer, New York, 1981.

Robert Boyer: Department of Mathematics, Drexel University, Philadelphia, PA 19104, USA

E-mail address: rboyer@math.drexel.edu 


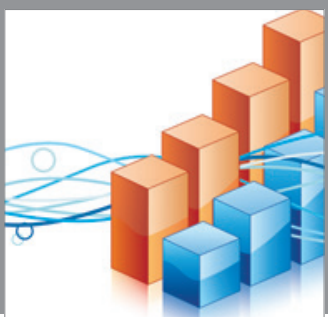

Advances in

Operations Research

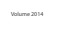

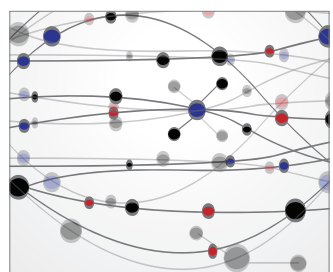

\section{The Scientific} World Journal
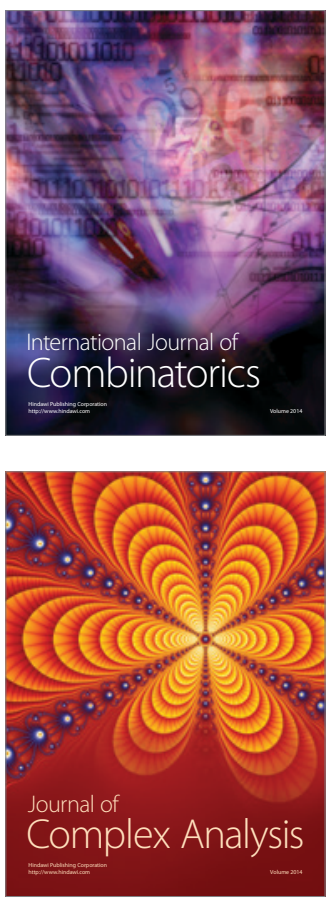

International Journal of

Mathematics and

Mathematical

Sciences
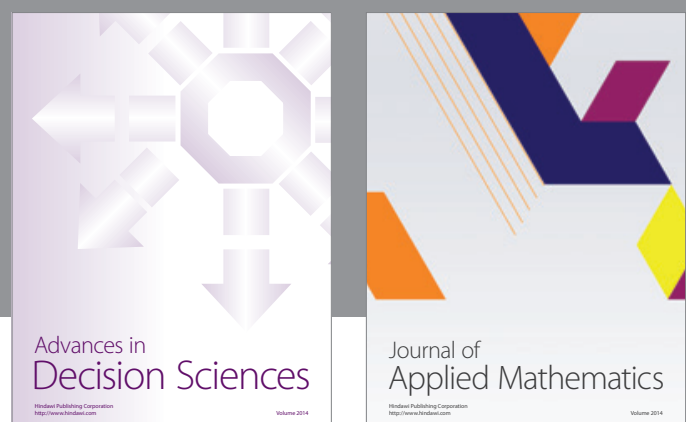

Journal of

Applied Mathematics
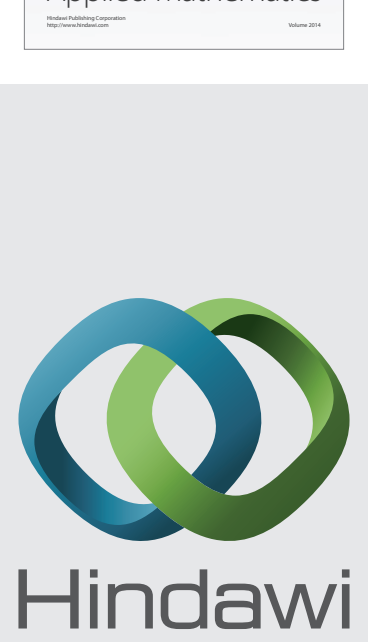

Submit your manuscripts at http://www.hindawi.com
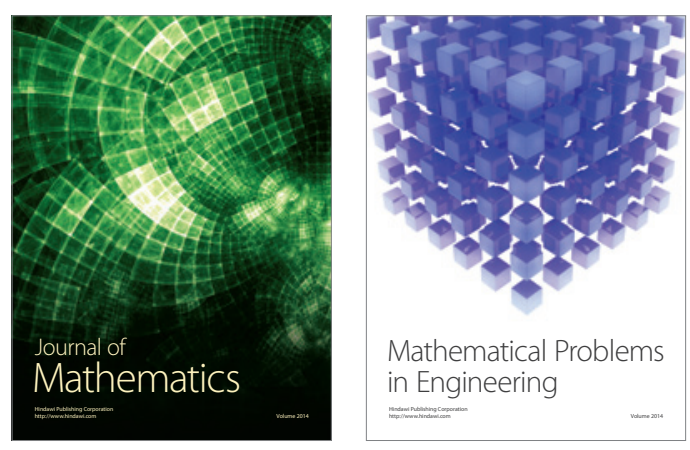

Mathematical Problems in Engineering
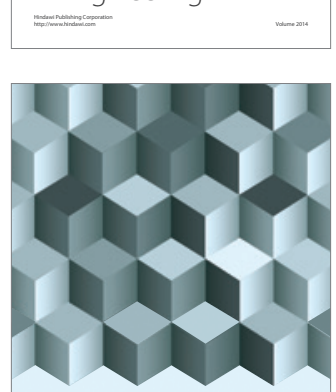

Journal of

Function Spaces
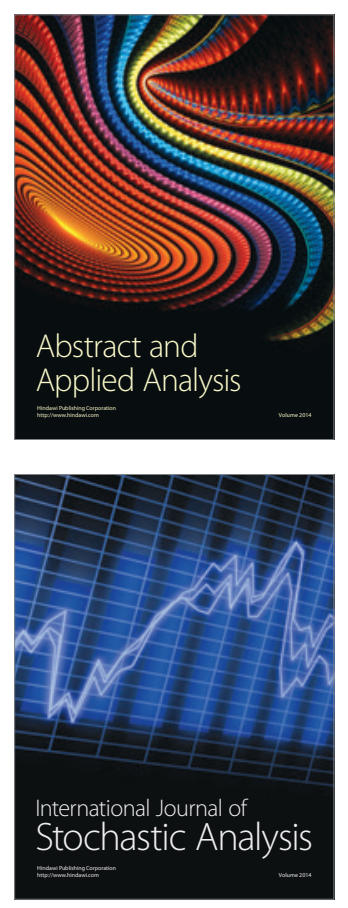

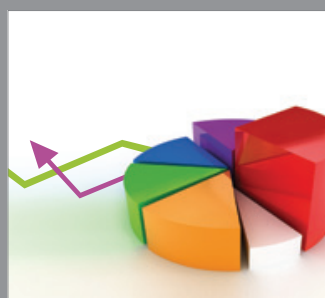

ournal of

Probability and Statistics

Promensencen
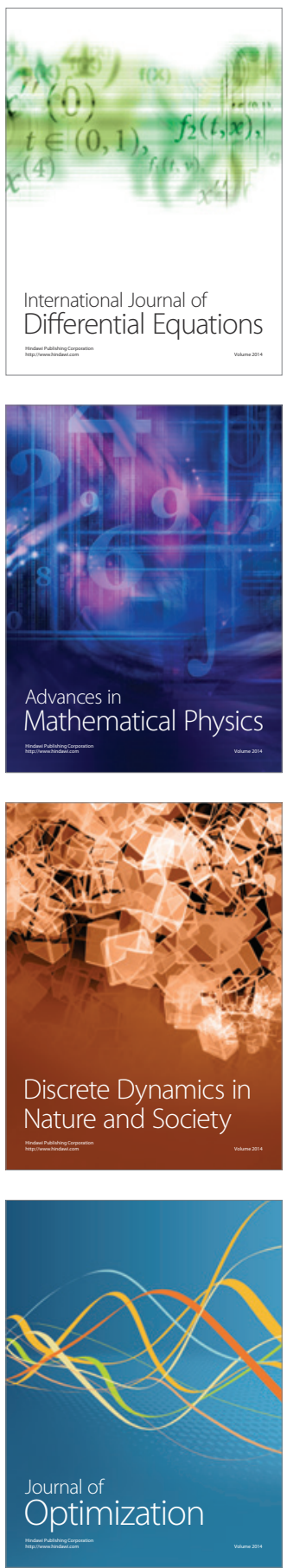